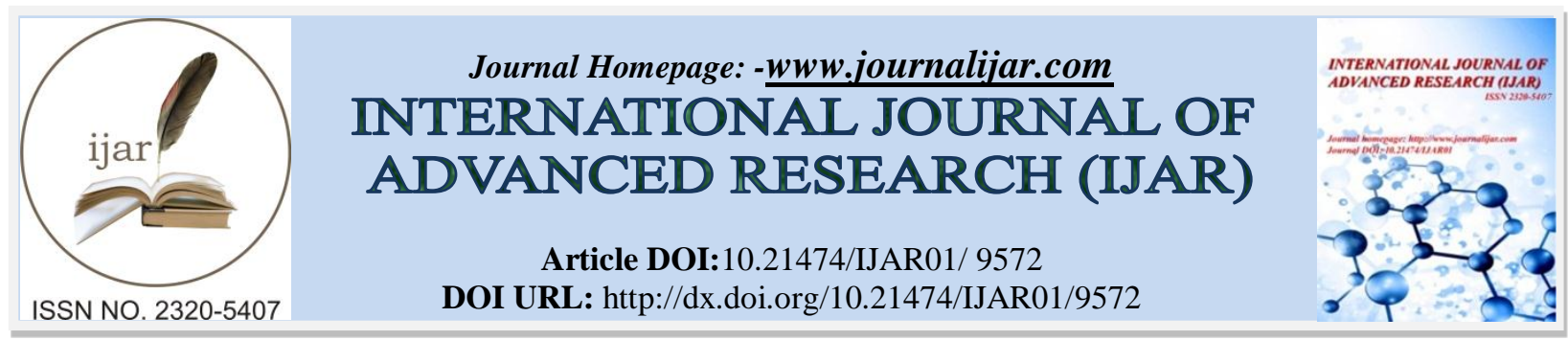

RESEARCH ARTICLE

\title{
CARDIOVASCULAR AUTONOMIC NEUROPATHY IN TYPE 2 DIABETES MELLITUS.
}

\section{Deepti P. Deshmukh ${ }^{1}$ and Govind Lone ${ }^{2}$.}

1. Associate Professor, Department of Medicine, Indira Gandhi Government Medical College, Nagpur, Maharashtra, India.

2. Ex-Resident, Department of Medicine, Indira Gandhi Government Medical College, Nagpur, Maharashtra, India.

\section{Manuscript Info}

Manuscript History

Received: 14 June 2019

Final Accepted: 16 July 2019

Published: August 2019

Key words:-

Cardiac autonomic neuropathy, Type 2 diabetes.

\section{Abstract}

Aim:To determine the frequency and severity of cardiovascular autonomic neuropathy in patients with type 2 diabetes mellitus and to assess the association of cardiovascular autonomic neuropathy with age, duration of diabetes, body mass index, hypertension, diabetic control $\left(\mathrm{Hb}_{\mathrm{Alc}} \%\right)$, lipid profile parameters and diabetic microangiopathy.

Material \&Methods: This hospital based cross-sectional study was carried out from November 2015 to October 2017.One hundred cases of Type 2 diabetes mellitus fulfilling inclusion, exclusion criteria were examined for detailed history, anthropometry, biochemical parameters and cardiovascular autonomic reflex tests suggested by Ewing and Clarke.

Conclusion: We observed that cardiovascular autonomic neuropathy was present in $42 \%$ cases and its presence had a statistically significant association with higher age, duration of diabetes, BMI, presence of hypertension, dyslipidemia and microangiopathy .

Copy Right, IJAR, 2019,. All rights reserved.

\section{Introduction:-}

Diabetes mellitus is a global epidemic affecting at least $8.3 \%$ of the global population and 371 million people all over the world (1). Of the microvascular complications in diabetes, cardiovascular autonomic neuropathy (CAN) is one of the commonest. CAN predisposes the patients to silent ischemia and sudden cardiac death and has a major impact on CVD morbidity and mortality (2). In spite of this, it is the most neglected and highly under-recognized complication. So early detection of CAN is of prime importance for risk stratification and intervention for preventing the serious consequences especially in diabetics (2). Hence this study was carried out with aim to determine the frequency and severity of cardiovascular autonomic neuropathy in patients with type 2 diabetes mellitus and to assess the association of cardiovascular autonomic neuropathy with age, duration of diabetes, body mass index, hypertension, diabetes control $\left(\mathrm{Hb}_{\mathrm{Alc}} \%\right)$, and lipid profile.

This hospital based cross-sectional study was carried out from November 2015 to October 2017. 100 cases of Type 2 diabetes mellitus in age group of $>30$ to 65 years were enrolled after satisfying inclusion and exclusion criteria.

Corresponding Author:-Deepti P. Deshmukh.

Address:-Associate Professor, Department of Medicine, Indira Gandhi Government Medical College,

Nagpur, Maharashtra, India. 
Thorough history was taken and examination was done with special emphasis on anthropometry and blood pressure and fundus examination. Fasting \& postprandial blood sugar levels, $\mathrm{Hb}_{\mathrm{Alc}}$, and lipid profile estimation was done. These cases were then subjected to five noninvasive cardiac autonomic function test as recommended by Ewing and Clarke. On the basis of abnormal test cases categorized into a). Normal b) Early CAN c) Definite CAN d) Severe CAN. These cases on the basis of cardiovascular autonomic neuropathy were divided into two groups- one with Type 2 DM with CAN and other Type 2 DM without CAN. These two groups were compared for age, duration and control of diabetes $\left(\mathrm{Hb}_{\mathrm{Alc}} \%\right)$, BMI, hypertension, dyslipidemia, presence of retinopathy, peripheral neuropathy, nephropathy. Institutional ethical committee approved this study. Informed consent was obtained prior to enrolling all subjects in the study.

The categorical variables were compared using the chi-square test. Odd's ratios (OR) and $95 \%$ confidence intervals were computed wherever applicable. Student $t$ test was used to calculate mean variables. A p-value of $<0.05$ was considered statistically significant. Multivariate analysis was carried out to study the independent association of cardiovascular autonomic neuropathy with different variables. Data was entered and analysed using the Strata software package version13.0.

\section{Observations}

Out of 100 cases, $56 \%$ were male. Maximum number of cases 37(37\%) were present in 41-50 years age group, in $51 \%$ of cases the duration of diabetes mellitus was in range of $1-5$ years while in (21\%) it was 6-10 years.Seven $(7 \%)$ had of diabetes mellitus of $>10$ years duration. Mean duration of diabetes mellitus was 4.3 years with SD 8.70. Out of 100 cases physical inactivity was present in $48(48 \%)$ cases, family history of hypertension and /or premature heart disease were present in $36(36 \%$ ) cases, hypertension in 44(44\%), abnormal waist to hip ratio was present in 29 cases and dyslipidemia was present in $71 \%$ cases.

Cardiovascular autonomic neuropathy was diagnosed by Ewing's criteria was seen in $42 \%$. Parasympathetic tests were positive in $42 \%$ cases of which Heart Rate response to deep breathing in $32 \%$ cases, Lying-to standing heart rate response in $24 \%$ and Valsalva maneuver in $11 \%$ cases were positive. Sympathetic tests were positive in $24 \%$ cases of which blood pressure response to change in posture was seen in $18 \%$ cases and diastolic blood pressure response to sustained hand grip was positive in 10(10\%) cases. Of these 100 cases, cardiovascular autonomic neuropathy was absent in 58\% cases, early (0.5-1.5) CAN was seen in $28 \%$ cases, definite CAN was seen in $9 \%$ cases and severe CAN was seen in 5\% (Figure 1)

Mean age in cases with CAN was 56.9 years (SD 7.26,range 40-65) which was higher than those in cases without CAN in which mean age was 49.53 years (SD 8.91, range 31-65). This difference was statistically significant (tstatistics $4.471, \mathrm{p}=0.00002$ ).

Mean duration of diabetes mellitus in cases with CAN was 6.42 years (SD 4.84,range 0.16-18) which was higher than those cases without CAN. This difference was statistically significant.(t-statistics $4.421, \mathrm{p}=0.00002$ )

Out of 42 cases with cardiac autonomic neuropathy 23(54.76\%) had BMI more than or equal to 25, whereas 19 (45.23\%) of 42 cases had BMI less than 25.Of 58 cases without cardiac autonomic neuropathy $09(15.51 \%)$ had BMI $\geq 25$ and $49(84.49 \%)$ of 58 cases had BMI $<25$. This difference was statistically significant(Odd ratio $=6.449$, confidence interval $2.56-17.18$, chi square $=17.24, \mathrm{p}=0.000016$ ).

Of 42 cases with CAN, 27(64.28\%) had hypertension, whereas 15 (35.71\%) of 42 cases were non-hypertensive. Of 58 cases without cardiac autonomic neuropathy $17(29.31 \%)$ had hypertension and $41(70.68 \%)$ of 58 cases were nonhypertensive. This difference was statistically significant. (Odds ratio=4.272, confidence interval 1.84-10.23, chisquare $=12.09, \mathrm{p}=0.0005)$.

Mean $\mathrm{Hb}_{\mathrm{Alc}}(\%)$ in cases with cardiac autonomic neuropathy was 9.36\%(SD 1.94,range 5.8-13.2) which was higher than those cases without cardiac autonomic neuropathy in which mean $\mathrm{Hb}_{\mathrm{Alc}}(\%)$ was $8.57 \%$ (SD 2.50, range 4.914.9).This difference was statistically non-significant.(t-statistics $1.7082, \mathrm{p}=0.09(>0.05$ no significant). The association of CAN with dyslipidemia and microvascular complications in diabetes has been tabulated as in table no 13 and 14 respectively. 
On multiple logistic regression $\mathrm{CAN}$ as dependent variable and age(in years), duration of DM(in months), hypertension , $\mathrm{BMI}(>25)$, serum triglyceride( $>150 \mathrm{mg} \%)$, serum $\mathrm{HDL}(\mathrm{M}<40 \mathrm{mg} \%$, F $<50 \mathrm{mg} \%)$, and serum $\operatorname{LDL}(>100 \mathrm{mg} \%)$ as independent variables in module 1 duration of $\mathrm{DM}(\mathrm{p}=0.12), \mathrm{BMI}(\mathrm{p}=0.04)$, and serum triglyceride $(\mathrm{p}=0.009)$ found statistically significant. (Table 1).

In module $2 \& 3$ duration of DM, and serum triglyceride were found statistically significant, whereas BMI was not $\operatorname{significant}(\mathrm{p}=0.06)$.

\section{Discussion:-}

In our study, out of 100 cases cardiovascular autonomic neuropathy was absent in 58(58\%) cases whereas $28(28 \%)$ cases had early (0.5-1.5) CAN, 9(9\%) cases had definite CAN and 5(5\%) had severe CAN.(table 6)

Mathur CP et al(3) reported absent CAN in $42 \%$, early changes in $20 \%$, definite in $30 \%$, severe in $4 \%$ and Atypical CAN in 4\%. Pillai JN et al (4) found that $21(42 \%)$ had severe CAN and $12(24 \%)$ had early CAN.

Mean age in our study in cases with cardiac autonomic neuropathy was 56.90 years (SD 7.26,range 40-65) .In Sinha et al.(5)study, mean age+/-SD of CAN present cases was $54.97 \pm 8.05$ and CAN absent was $52.66 \pm 3.29$ $(\mathrm{p}=0.076)$ and its significance on multilogistic analysis similar to our study. Gulichsen et al.(6) Anca et al.(7) found that cardiovascular autonomic neuropathy was associated with higher mean age..

In our study $27(64.28 \%$ ) of 42 cases with cardiac autonomic neuropathy had hypertension whereas

Gulichsen et al.(6) found that cardiovascular autonomic neuropathy was associated with higher systolic blood pressure. In study by Sinha et al.(5) mean SBP (mmHg) $160 \pm 12.95$ (with CAN) and $155.5 \pm 14.9$ (without CAN) $\mathrm{p}=0.0952$ found significant. Anca et al(7)found significant association $(\mathrm{p}=0.004)$ while mean $\mathrm{DBP}(\mathrm{mmHg}) 100 \pm$ 9.43(with CAN) $97.67 \pm 12.62$ (without CAN) p=0.2805 was not found significant.

In our study microvascular complications of type 2 diabetes-Background retinopathy ( $\mathrm{p}=0.002)$,Diabetic nephropathy $(\mathrm{p}=0.00026)$ and peripheral neuropathy $(\mathrm{p}=0.000001)$ all were associated with cardiovascular autonomic neuropathy.

Gulichsen et al.(6) found that Patients with CAN had more nephropathy and retinopathy, and a higher vibration threshold(peripheral neuropathy). Anca et al.(7) studied retinopathy $(\mathrm{p}=0.001)$, eGFR $(0.001)$ and peripheral neuropathy(0.001) in cases with and without cardiovascular autonomic neuropathy and found to be significant.

\section{Conclusion:-}

This hospital based cross-sectional study was carried out from November 2015 to October 2017.

One hundred cases of Type 2 diabetes mellitus fulfilling inclusion, exclusion criteria and willing to give written informed consent were enrolled from the diabetes specialty clinic, medicine outpatient department or from those admitted to medicine wards of tertiary care centre.

Cases were examined for detailed history anthropometry, biochemical parameters and cardiovascular autonomic reflex tests suggested by Ewing and Clarke.

They were also subjected to carotid intima media thickness measurement.

Following are the conclusions of the study.

1. Frequency of cardiovascular autonomic neuropathy (CAN) was present in $42(42 \%)$ cases out of 100 cases studied. Higher number of cases had Early CAN (28\%) followed by definite CAN (9\%) and Severe CAN (5\%) of the total cases.

2. Of the Ewing's cardiovascular autonomic function tests, parasympathetic tests (of which specifically heart rate response to deep breathing) were positive in higher number of cases (42\%) than sympathetic tests (24\%).

3. Cardiovascular autonomic neuropathy was associated with higher mean age $(\mathrm{p}=0.00002)$, higher mean duration of diabetes (0.00002), higher BMI(0.00001) and presence of hypertension(0.0005) in cases and this association was statistically significant, whereas there was no association of CAN with control of diabetes as assessed by $\mathrm{HB}_{\mathrm{A} 1 \mathrm{c}} \%(0.09)$. 
4. Of lipid parameters there was statistically significant association of CAN with high serum triglycerides $(\mathrm{p}=0.0001)$, high serum $\operatorname{LDL}(0.0044)$ and low serum $\operatorname{HDL}(\mathrm{p}=0.0237)$ however only high serum cholesterol $(\mathrm{p}=0.392)$ didn’t show statistically significant association.

There was statistically significant association of CAN with all three microvascular complications of type 2 diabetes

We observed that cardiovascular autonomic neuropathy had statistically significant association with higher age , duration of diabetes, BMI ,presence of hypertension, dyslipidemia(except total cholesterol)and all three microvascular complications while CAN was not associated with control of diabetes this may be due to small sample size. On multiple logistic regression CAN was associated with duration of DM. serum triglyceride. There was no association seen with background retinopathy this may be due to we have included only preproliferative retinopathy excluding proliferative retinopathy or may be due to small sample size.

\section{Distribution of cases according to Cardiovascular autonomic neuropathy severity \\ - Absent(0) $\quad$ 1)Early(0.5-1.5) $\quad$ 2)Definite(2-3) $\quad$ 3)Severe(>3.5)}

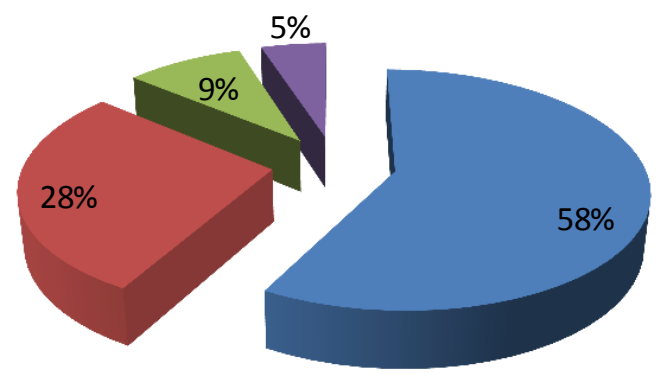

Figure 1.diagram showing distribution of cases according to cardiovascular autonomic neuropathy severity.

Table1:MULTIPLE LOGISTIC REGRESSION WITH CARDIOVASCULAR AUTONOMIC NEUROPATHY AS DICHOTOMOUS DEPENDANT VARIABLE AND AGE,DURATION OF DM,HYPERTENSION,BMI,SERUM TRIGLYCERIDES,SERUM HDL,SERUM LDL AS INDEPENDANT VARIABLES

\begin{tabular}{|l|l|l|l|}
\hline INDEPENDENT VARIABLES & ODD'S RATIO & $95 \%$ CI & P VALUE \\
\hline AGE(in years) & 1.054 & 0.9671 .147 & 0.22 \\
\hline DURATION OF DM(in months) & 1.018 & $1.004-1.0333$ & 0.012 \\
\hline HYPERTENSION & 2.622 & $0.776-8.850$ & 0.12 \\
\hline BMI $(\geq 25)$ & 1.499 & $1.012-2.220$ & 0.04 \\
\hline $\begin{array}{l}\text { SERUM } \\
\text { TRIGLYCERIDES( } \geq 150 \mathrm{mg} \%)\end{array}$ & 1.017 & $1.004-1.030$ & 0.009 \\
\hline $\begin{array}{l}\text { SERUM HDL }(\mathrm{M}<40 \mathrm{mg} \%, \\
\text { F }<50 \mathrm{mg} \%)\end{array}$ & 0.972 & $0.933-1.013$ & 0.18 \\
\hline SERUM LDL $(\geq 100 \mathrm{mg} \%)$ & 0.992 & $0.970-1.013$ & 0.48 \\
\hline
\end{tabular}

\section{Implications}

Frequency of cardiovascular autonomic neuropathy is high in diabetic cases. CAN predisposes the patients to silent ischemia and sudden cardiac death and has a major impact on CVD morbidity and mortality. In spite of this, it is the most neglected and highly under-recognised complication, so early diagnosis and therapeutic interventions will help in preventing both morbidity and mortality. 


\section{References:-}

1. Federation ID. IDF Diabetes Atlas 2012 Update. 2012

2. R. E. Maser, B. D. Mitchell, A. I. Vinik, and R. Freeman, "The association between cardiovascular autonomic neuropathy and mortality in individuals with diabetes a meta-analysis," Diabetes Care, vol. 26, no. 6, pp. 18951901, 2003.

3. Mathur CP, Gupta D. QTC prolongation in diabetes mellitus - an indicator of cardiac autonomic neuropathy. J Indian academy of clinical medicine. 2006; 7(2):130-32.

4. Pillai JN, Madhavan S. Cardiac Autonomic Neuropathy and QTc Interval in Type 2 Diabetes. Heart India. 2015;3:8.

5. Sinha PK, Santra G, De D, Saha A, Biswas K, Bhattacharya P, Ghosh P. Carotid intima-media thickness un type 2 diabetes mellitus patients with cardiac autonomic neuropathy. J Assoc Physicians India 2012;60:14-18

6. Elisabeth Gulichsen, D.M.Sc.,1 Jesper Fleischer, M.Sc.B.M.E.,2 Niels Ejskjaer, D.M.Sc. Ph.D.,2 Ebbe Eldrup, D.M.Sc.,3 and Lise Tarnow, D.M.Sc.1 "Screening for Diabetic Cardiac Autonomic Neuropathy Using a New Handheld Device" Journal of Diabetes Science and Technology July 2012,Volume 6, Issue 4

7. Anca Moususianu,1,2 Rodica BslaGa,1 Septimiu Voidszan,3 and Zoltán Bajkó1 "Cardiovascular Autonomic Neuropathy in Context of Other Complications of Type 2 Diabetes Mellitus" Hindawi Publishing Corporation BioMed Research International, Volume 2013, Article ID 507216, 8 pages, http://dx.doi.org/10.1155/2013/507216. 